\title{
Comunicación asertiva para mejorar el desarrollo institucional en la unidad educativa Juan Bautista Aguirre
}

\section{RESUMEN}

\author{
Wendy Yesenia Alvarado Torres \\ P7001249969@ucvvirtual.edu.pe \\ https://orcid.org/0000-0002-8133-8577 \\ Universidad César Vallejo-Perú \\ Nancy Aracely Castro Ramírez \\ P7001249979@ucvvirtual.edu.pe \\ https://orcid.org/0000-0003-3157-6320 \\ Universidad César Vallejo-Perú \\ David Armando Mondragón Sánchez \\ damondragonm@ucvvirtual.edu.pe \\ https://orcid.org/0000-0002-2025-3377 \\ Universidad César Vallejo-Perú \\ Johanna Del Carmen Romero Jiménez \\ P7001254326@ ucvvirtual.edu.pe \\ https://orcid.org/0000-0001-8733-8191 \\ Universidad César Vallejo-Perú
}

La investigación precisó como fin plantear un modelo de comunicación asertiva entre autoridades y docentes con base en la teoría de Fernández para mejorar el desarrollo institucional de la Unidad Educativa Juan Bautista Aguirre del cantón Daule, provincia del Guayas, 2020. La metodología en que se basó el estudio ha sido de tipo aplicado, con un enfoque cuantitativo; y diseño no experimental de alcance transaccional, se hizo uso de una muestra censal de 72 participantes, los datos fueron compilados por medio de la técnica de la encuesta usando un cuestionario para las variables bajo análisis, las interrogantes de los cuestionarios fueron revisadas según estimación de profesionales en el área para después hacer la prueba de fiabilidad del Alfa de Cronbach con un coeficiente de 0,951 para la variable comunicación asertiva y 0,936 para la variable desarrollo institucional; en consecuencia, los instrumentos aplicados son altamente fiables. Efectuada la prueba de hipótesis por medio de la prueba no paramétrica U Mann-Whitney se determinó que diseñar un modelo de comunicación asertiva mejorará el desarrollo institucional en la Unidad Educativa Juan Bautista Aguirre.

Palabras clave: comunicación asertiva; desarrollo institucional; autoridades; docentes. 


\title{
Assertive communication to improve institutional development in the Juan Bautista Aguirre educational unit
}

\begin{abstract}
The purpose of the research was to propose an assertive communication model between authorities and teachers based on Fernández's theory to improve the institutional development of the Juan Bautista Aguirre Educational Unit in Daule, Guayas province, 2020. The methodology on which it was based the study has been of an applied type, with a quantitative approach; and non-experimental design with a transactional scope, a census sample of 72 participants was used, the data was compiled by means of the survey technique using a questionnaire for the variables under analysis, the questions of the questionnaires were revised according to the estimation of professionals in the area and then made the Cronbach's Alpha reliability test with a coefficient of 0.951 for the assertive communication variable and 0.936 for the institutional development variable; consequently, the applied instruments are highly reliable. Once the hypothesis test was carried out using the nonparametric U Mann-Whitney test, it was determined that designing an assertive communication model will improve institutional development in the Juan Bautista Aguirre Educational Unit.
\end{abstract}

Key words: assertive communication; institutional development; authorities; teachers.

Artículo recibido: 18. Julio. 2021

Aceptado para publicación: 08. Agosto. 2021 Correspondencia: P7001249969@ucvvirtual.edu.pe Conflictos de Interés: Ninguna que declarar 


\section{INTRODUCCIÓN}

A nivel mundial la comunicación asertiva representa un rol crucial por su protagonismo en el desarrollo institucional de las unidades educativas; la Organización de las Naciones Unidas para la Educación, la Ciencia y la Cultura ([UNESCO] , 2011) conocedor de este influjo insiste que la comunicación es la destreza de impulsar y sostener pláticas para generar un ambiente que permita a las autoridades y docentes concebir actividades que ayuden en lograr el desarrollo de la institución; por ende, es necesario distinguir a la institución como una entidad y el lenguaje como formador de vías comunicacionales.

Briones, Urquiza, Navia \& Guevara (2020) percibieron a la comunicación asertiva como un modelo para optimizar prácticas que inicien, favorezcan y desarrollen las relaciones interpersonales en diferentes etapas a través del tiempo; además, en una institución educativa quienes deben fomentar una correcta manera de comunicarse recae sobre las autoridades; Rojas, Dolibeth, Mota \& Quiñonez (2020) aseveran que un líder es quien desempeña preponderancia sobre sus subalternos, es la persona que orienta a los demás para alcanzar un objetivo en específico con el propósito de que todos se sientan comprometidos en cada parte del desarrollo del proceso, en consecuencia, en una unidad educativa quienes personifican la autoridad institucional son ejemplo de cómo se debe empezar y fortalecer la comunicación asertiva entre estos y los docentes; según Education Week (2019) las autoridades y docentes deben apoyar en establecer vínculos de compañerismo; sin embargo, los resultados que obtuvieron en la encuesta que aplicaron, mostraron que el más fuerte impedimento para que esto se lleva a cabo radica del lado de las autoridades; consecuentemente, son estos los que habrán de trabajar con mayor empeño en modificar el modo de comunicación que adoptan con sus compañeros docente. Como base para mejorar cualquier institución, el talento humano es quien realiza los procesos básicos que se ejecutan para lograr el desarrollo institucional, esto es posible si existe entre todos los miembros el compromiso de apoyar los cambios necesarios para alcanzar este fin; según Pardo \& Díaz (2014) declaran que las perspectivas frente a hechos negativos y las restricciones en la comunicación son posibles obstáculos que demoran o entorpecen el desarrollo institucional; la comunicación asiste en la ejecución de cada proceso de la actividad humana, no es simplemente platicar, lo primordial es saber escuchar para que prepondere la comunicación asertiva, este comportamiento favorece al ámbito institucional, logrando que los individuos adquieran y dominen una comunicación 
apropiada para interrelacionarse con un grupo dinámico estimulando el desarrollo institucional.

La Unidad Educativa Juan Bautista Aguirre del cantón Daule no está apartada de este contexto y periódicamente las autoridades y docentes se ven comprometidos en escenarios en la que la comunicación es discrepante; una comunicación apática o sarcástica entre estos actores, repercute de modo negativo en la motivación docente ya sea para realizar sus actividades académicas u otra diligencia encargada por la autoridad, fenómeno notorio en la carencia de empeño al organizar las clases o apatía de contribuir con soluciones relacionadas al desarrollo institucional.

Por todo lo expuesto el objetivo general del estudio busca proponer un modelo de comunicación asertiva entre autoridades y docentes basado en la teoría de Fernández para mejorar el desarrollo institucional de la Unidad Educativa Juan Bautista Aguirre del cantón Daule, provincia del Guayas, 2020 y como hipótesis de investigación el diseño de un modelo de comunicación asertiva basado en la teoría de Fernández mejorará el desarrollo institucional en la Unidad Educativa Juan Bautista Aguirre del cantón Daule, 2020.

\section{ESTRATEGIAS METODOLÓGICAS O MATERIALES Y MÉTODOS}

La investigación se realizó bajo un enfoque cuantitativo que para Daniel (2016) la mayor superioridad de este enfoque es el uso de la estadística como herramienta para ahorrar recursos y tiempo; además de concentrarse en probar hipótesis y teorías.

La inclinación de esta investigación fue aplicada de tipo descriptivo, que para Marotti \& Pedroso (2018) declaran que una investigación que pretende solucionar inconvenientes en un entorno determinado se llama investigación aplicada, mientras que para Atmowardoyo (2018) una investigación de tipo descriptivo detalla los fenómenos observados con la mayor fidelidad posible; es otras palabras tiene como propósito pormenorizar de manera exacta y sistemática un entorno o fenómeno de una población y al mismo tiempo hace uso de una extensa variedad de métodos de investigación para comparar una o más variables.

Esta investigación fue de un diseño no experimental que para Thomas (2016) está desprovisto de cualquier injerencia sobre la variable independiente, esta se cimenta en el estudio de fenómenos que no hayan sufrido ningún tipo de intervención para reflexionar con posterioridad. 
La metodología tuvo un alcance transaccional, que para Zangirolami, Echeimberg \& Leone (2018) es muy ventajoso en estudios descriptivos ya que se emplea en estudios que son de índole analítico y las derivaciones que se consigan deben ser dilucidados por investigadores con experiencia en ese ámbito de estudio en específico.

La población para Majid (2018) es el grupo de interés que representa el propósito del estudio que se pretende investigar o mostrar, para esta investigación la población fue constituida por cinco autoridades y sesentaisiete docentes que trabajan en la oferta educativa ordinaria de la Unidad educativa Juan Bautista Aguirre del cantón Daule.

Para dar una real visión institucional se contempló una muestra censal que para Ávila (2019) es cuando se considera cada uno de los elementos poblacionales para la ejecución de la investigación; además se consideró un muestreo probabilístico aleatorio simple. Como técnica se utilizó la encuesta; y se define según Ponto (2015) como el ejercicio de reunir información de una establecida cantidad de personas a través de contestaciones a interrogantes efectuadas por un encuestador.

El instrumento con la intención de mostrar resultados apegados al propósito de esta investigación fue un cuestionario que según Mitchell, Loew, Millington \& Dale (2016) es una secuencia de interrogantes que podrían estar estructuradas para responder de modo abierto o cerrado; el instrumento utilizado tuvo una distribución de escala de medición ordinal que para Arvidsson (2019) posee valores con un orden específico, pero no se sabe la distancia entre estos; simplifica un rango determinado de respuestas a una explícita pregunta o aseveración;

También se realizó una prueba piloto con 15 partícipes para realizar la prueba de fiabilidad de los instrumentos aplicándose el Alfa de Cronbach donde se obtuvo para la variable comunicación asertiva un coeficiente de 0,951 y para la variable desarrollo institucional 0,936 obteniéndose que los instrumentos son altamente fiables. Para el cotejo de los datos se utilizó la estadística descriptiva e inferencial.

En cumplimiento de los aspectos éticos que todo investigador debe considerar para la realización de cualquier estudio, se contemplaron los principios básicos de la beneficencia, la no maleficencia, la autonomía y la justicia con la visión de aportar soluciones con la investigación realizada, respetando las normas de conducta ética que tienen que ser considerados en el campo de la ciencia.

\section{RESULTADOS Y DISCUSIÓN}




\section{Tabla 1}

Nivel de comunicación asertiva y desarrollo institucional.

\begin{tabular}{lcccccccc}
\hline \multirow{2}{*}{ Categorías } & \multicolumn{3}{c}{ Comunicación asertiva } & \multicolumn{3}{c}{ Desarrollo institucional } \\
\cline { 2 - 9 } & \multicolumn{2}{c}{ Autoridades } & \multicolumn{2}{c}{ Docentes } & Autoridades & \multicolumn{2}{c}{ Docentes } \\
\cline { 2 - 9 } & $\mathbf{f}$ & $\mathbf{\%}$ & $\mathbf{f}$ & $\mathbf{\%}$ & $\mathbf{f}$ & $\mathbf{\%}$ & $\mathbf{f}$ & $\mathbf{\%}$ \\
\hline Deficiente & 0 & 0 & 0 & 0 & 2 & 3 & 2 & 3 \\
Regular & 0 & 0 & 0 & 0 & 38 & 57 & 33 & 49 \\
Bueno & 5 & 100 & 5 & 100 & 27 & 40 & 32 & 48 \\
\hline Total & $\mathbf{5}$ & $\mathbf{1 0 0}$ & $\mathbf{5}$ & $\mathbf{1 0 0}$ & $\mathbf{6 7}$ & $\mathbf{1 0 0}$ & $\mathbf{6 7}$ & $\mathbf{1 0 0}$ \\
\hline
\end{tabular}

Nota. Datos obtenidos en la encuesta. Elaborado por: Wendy Yesenia Alvarado Torres.

El 100\% de las autoridades de la Unidad Educativa Juan Bautista Aguirre, opinan estar en un nivel bueno de comunicación asertiva; el 3\% de los docentes opinan que existe un nivel deficiente en comunicación asertiva; sienten que no existe un adecuado modo de comunicación, el $49 \%$ de los docentes opinan que existe un nivel regular en comunicación asertiva, creen necesario que se realice mejoras en la comunicación y un $48 \%$ de los docentes opinan que existe un nivel bueno en comunicación asertiva, creen que la comunicación existente es la apropiada. En observancia de las derivaciones que se obtuvieron Calderón (2017) recalca la eficacia de interceder en los problemas entre autoridades y docentes valiéndose de la comunicación asertiva para relacionarse con avenencia y comprensión con cada uno de los integrantes de la comunidad educativa.

También Fernández (2016) advierte que cuando existe moderación en la declaración de los sentimientos, las relaciones con terceros ya sea en un entorno privado o laboral serán las apropiadas para la creación de un ámbito agradable para todos, además el tener un modelo determinado de comunicación facilita la aplicación de comportamientos y destrezas adecuadas que fijen sensatez en el modo de comunicarse y como resultado se obtendrá una opción más agradable y admitida de interrelación.

El 100\% de las autoridades de la Unidad Educativa Juan Bautista Aguirre, opinan estar en un nivel bueno de desarrollo institucional; el 3\% de los docentes de la Unidad Educativa Juan Bautista Aguirre opinan que existe un nivel deficiente de desarrollo institucional; sienten que es necesario que se efectúen reuniones con docentes de otras instituciones para reforzar experiencias y mejorar el servicio brindado; el 57\% de los docentes opinan que existe un nivel regular de desarrollo institucional, observan que las autoridades no organizan actividades de mejora para los docentes que no alcanzan los 
requerimientos mínimos en su desempeño pedagógico en aula; por último, el $40 \%$ de los docentes opinan que existe un nivel bueno de desarrollo institucional, ya que las autoridades realizan sondeos en los procesos internos de la institución. En concordancia a los resultados obtenidos Angamarca (2016) expone la necesidad de un perenne mejoramiento contemplando la presencia de fuerzas intrínsecas y extrínsecas que inciden en el desarrollo institucional;

Igualmente, Leiva \& Astorga (2014) aseveran que el desarrollo institucional es el efecto de realizar una transformación en su totalidad, valiéndose de métodos prácticos en razón de las distintas contribuciones que implicados directos o indirectos plantean para confrontar los diferentes conflictos que puedan suscitar en las instituciones.

\section{Tabla 2}

Categorización de frecuencias de las dimensiones de la variable comunicación asertiva.

\begin{tabular}{|c|c|c|c|c|c|c|c|c|c|c|c|c|}
\hline \multirow{3}{*}{$\begin{array}{c}\text { Categoría } \\
\mathrm{s}\end{array}$} & \multicolumn{4}{|c|}{ Asertividad laboral } & \multicolumn{4}{|c|}{ Comunicación abierta } & \multicolumn{4}{|c|}{$\begin{array}{c}\text { Apreciación de la } \\
\text { convivencia }\end{array}$} \\
\hline & \multicolumn{2}{|c|}{ Autoridades } & \multicolumn{2}{|c|}{ Docentes } & \multicolumn{2}{|c|}{ Autoridades } & \multicolumn{2}{|c|}{ Docentes } & \multicolumn{2}{|c|}{ Autoridades } & \multicolumn{2}{|c|}{ Docentes } \\
\hline & $f$ & $\%$ & $f$ & $\%$ & $f$ & $\%$ & $\mathrm{f}$ & $\%$ & $\mathrm{f}$ & $\%$ & $\mathrm{f}$ & $\%$ \\
\hline Deficiente & 0 & 0 & 10 & 15 & 0 & 0 & 2 & 3 & 0 & 0 & 1 & 2 \\
\hline Regular & 0 & 0 & 30 & 45 & 0 & 0 & 34 & 51 & 1 & 20 & 34 & 51 \\
\hline Bueno & 5 & 100 & 27 & 40 & 5 & 100 & 31 & 46 & 4 & 80 & 32 & 47 \\
\hline Total & 5 & 100 & 67 & 100 & 5 & 100 & 67 & 100 & 5 & 100 & 67 & 100 \\
\hline
\end{tabular}

Nota. Datos obtenidos en la encuesta. Elaborado por: Wendy Yesenia Alvarado Torres.

El 100\% de las autoridades de la Unidad Educativa Juan Bautista Aguirre, opinan que brindan un nivel bueno de asertividad laboral; mientras un $15 \%$ de los docentes opinan que existe un nivel deficiente, ya que sienten que las autoridades no les ofrecen las mismas oportunidades como a otros compañeros; además el $57 \%$ opinó que existe un nivel regular debido a que conciben que las autoridades no logran dar una alocución positiva cuando existen contratiempos; por último, sólo el $40 \%$ de los docentes opinan que existe un nivel bueno de asertividad laboral dentro de la institución educativa. Estos resultados coinciden con Leal (2019) donde concibió que la escasa asertividad laboral de la institución donde efectuó su trabajo de investigación fueron evidentes los problemas entre los colaboradores de la unidad educativa demostrando la necesidad de optimizar la comunicación y así modificar lo que sea necesario para lograr un trabajo mancomunado, 
siendo las autoridades quienes impulsen un ambiente laboral grato para cada uno de los integrantes.

El 100\% de las autoridades de la Unidad Educativa Juan Bautista Aguirre, opinan que se encuentran en un nivel bueno de comunicación abierta; en tanto un 3\% de los docentes opinan que existe un nivel deficiente, ya que las autoridades no formulan claramente sus ideas; además el $51 \%$ opinó que existe un nivel regular debido al uso inadecuado de muletillas que obstaculizan un real entendimiento de las observaciones que pretenden compartir; por último, tan sólo el $46 \%$ de los docentes opinan que existe un nivel bueno de comunicación abierta por parte de las autoridades. Estos hallazgos concuerdan con Cortez (2018) que da notabilidad al alcance que autoridades como docentes deben dar al fortalecimiento de las competencias socioemocionales para conseguir un mejoramiento integral de la institución;

De igual manera, Corrales, Quijano \& Góngora (2017) también expusieron que las destrezas sociales amparan una extensa combinación de potencialidades que conectan las tácticas y estilos que favorecen una correcta comunicación.

El 80\% de las autoridades de la Unidad Educativa Juan Bautista Aguirre, opinan que se encuentran en un nivel bueno de la apreciación de la convivencia y un $20 \%$ en un nivel regular; en cambio los docentes en un $2 \%$ opinan que existe un nivel deficiente, ya que las autoridades no contribuyen en un buen clima dentro de la institución; además el $51 \%$ opinó que las autoridades no son muy propensos a una relación menos formal, el $47 \%$ de los docentes opinan que existe un nivel bueno de la apreciación de la convivencia entre autoridades y docentes. Las derivaciones obtenidas coinciden con Román (2016) donde asevera la importancia de saber sortear barreras comunicacionales que impidan o problematicen la comunicación para conseguir el fortalecimiento de la comunicación asertiva entre autoridades y docentes.

\section{Tabla 3}

Categorización de frecuencias de las dimensiones de la variable desarrollo institucional. 


\begin{tabular}{lcccccccc}
\hline & \multicolumn{3}{c}{ Ámbito humano } & \multicolumn{3}{c}{ Gestión directiva } \\
\cline { 2 - 9 } Categorías & \multicolumn{2}{c}{ Autoridades } & \multicolumn{2}{c}{ Docentes } & \multicolumn{2}{c}{ Autoridades } & \multicolumn{2}{c}{ Docentes } \\
\cline { 2 - 9 } & $\mathbf{f}$ & $\mathbf{\%}$ & $\mathbf{f}$ & $\mathbf{\%}$ & $\mathbf{f}$ & $\mathbf{\%}$ & $\mathbf{f}$ & $\mathbf{\%}$ \\
\hline Deficiente & 0 & 0 & 4 & 6 & 0 & 0 & 4 & 6 \\
Regular & 3 & 60 & 35 & 52 & 1 & 20 & 19 & 28 \\
\multirow{2}{*}{ Bueno } & 2 & 40 & 28 & 42 & 4 & 80 & 44 & 66 \\
\hline Total & $\mathbf{5}$ & $\mathbf{1 0 0}$ & $\mathbf{6 7}$ & $\mathbf{1 0 0}$ & $\mathbf{5}$ & $\mathbf{1 0 0}$ & $\mathbf{6 7}$ & $\mathbf{1 0 0}$ \\
\hline
\end{tabular}

Nota. Datos obtenidos en la encuesta. Elaborado por: Wendy Yesenia Alvarado Torres.

El 40\% de las autoridades de la Unidad Educativa Juan Bautista Aguirre, opinan que se encuentran en un nivel bueno en el ámbito humano y un $60 \%$ en un nivel regular; en cambio los docentes en un 6\% opinan que existe un nivel deficiente, ya que es necesario el aumento de cupo para la matriculación de nuevos estudiantes según la demanda por parte de la comunidad; además el 52\% calificó el ámbito humano en un nivel regular porque no se conciertan reuniones con docentes de otras instituciones para socializar experiencias y mejorar el servicio educativo, el $42 \%$ de los docentes opinan que existe un nivel bueno del ámbito humano. Estos resultados corroboran lo expuesto por Moscoso (2016) que discurrió que una exigua gestión institucional origina un entorpecimiento en el desarrollo de cualquier institución, deduciéndose que se debe procurar efectuar una planificación que induzca a una mejora continua; además Fabiani (2017) también declaró la significancia de contar con otras vivencias educativas por parte de servidores de otras instituciones con la finalidad de hallar soluciones generales aunque recalcó que cada institución es responsable de su desenvolvimiento.

El 80\% de las autoridades de la Unidad Educativa Juan Bautista Aguirre, opinan que se encuentran en un nivel bueno en la gestión directiva y un $20 \%$ en un nivel regular, en contraparte los docentes en un $6 \%$ opinan que existe un nivel deficiente, debido a que sólo a veces las autoridades informan acerca de las observaciones derivadas de las clases demostrativas que se efectúan para verificar el grado de desenvolvimiento en el aula por parte de los docentes, $28 \%$ calificó en un nivel regular ya que expusieron que no se llevan a cabo jornadas de reforzamiento para los docentes que presentaron problemas en las clases demostrativas; Guerra \& Vargas (2016) igualmente vieron necesario que para lograr el desarrollo de cualquier institución se precisa reformar procedimientos, técnicas y procesos que se realicen internamente; si se hacen estos correctivos se darán cambios que conduzcan al desarrollo, también Garbanzo (2016) manifestó que el desarrollo 
institucional supone del cumplimiento o mejoramiento de proyectos estratégicos específicos donde se delinea como carear las inconvenientes fehacientes que se observen en las instituciones.

El test de normalidad aplicados fue el estadístico Kolmogorov-Smirnov donde se comprobó que los datos presentan una distribución no normal ya que se obtuvo un nivel de significancia menor de 0,05 precisándose para la prueba de hipótesis la prueba no paramétrica U Mann-Whitney donde se obtuvo que el valor p de la variable comunicación asertiva fue de 0,004 y el de desarrollo institucional 0,032; aceptándose la hipótesis de investigación.

\section{CONCLUSIÓN O CONSIDERACIONES FINALES}

1) Un modelo de comunicación asertiva entre autoridades y docentes basado en la teoría de Fernández mejorará el desarrollo institucional en favor de toda la comunidad educativa.

2) Las características que mostró la comunicación asertiva entre autoridades y docentes en la Unidad Educativa Juan Bautista Aguirre del cantón Daule es que las autoridades no ofrecen equitativamente oportunidades ante petitorios de parte de los docentes, no adoptan una aptitud de tolerancia ante eventos inesperados, no disponen con fluidez verbal apropiada y, por último, sostienen un trato jerárquico preponderante con los docentes.

3) Al analizar los factores que presentó el desarrollo institucional en la Unidad Educativa Juan Bautista Aguirre del cantón Daule, se evidenció que el cupo establecido para la matriculación de los estudiantes nuevos no es equitativo según las necesidades de la comunidad; igualmente no se efectúan socializaciones con docentes de diferentes instituciones con la intención de transmitir experiencias para subsanar cualquier insuficiencia en el servicio educativo y para finiquitar la institución difícilmente efectúa proyectos que favorezcan al desarrollo de la comunidad; por el contrario, las autoridades calificaron el ámbito humano como buena en un $40 \%$ y la gestión directiva en un $80 \%$ como buena también.

4) La distribución de los datos fue no normal y se utilizó para la prueba de hipótesis el test U Mann-Whitney donde la comunicación asertiva reflejó un nivel de significancia de 0,004 y el desarrollo institucional 0,032 y, por lo tanto, se ratificó que un modelo 
de comunicación asertiva mejora el desarrollo institucional de la Unidad Educativa Juan Bautista Aguirre.

5) La propuesta fue estructurado acorde al análisis de la encuesta donde se reflejó una evidente discrepancia de las apreciaciones de las autoridades y docentes precisándose de contar con un modelo de comunicación asertiva, que contribuya en mejorar el desarrollo institucional en la Unidad Educativa Juan Bautista Aguirre del cantón Daule.

\section{REFERENCIAS BIBLIOGRAFICAS}

Angamarca, S. (2016). Diseño de Intervención en Desarrollo Institucional en la Universidad Católica de Cuenca, Sede Cañar [Tesis de maestría, Universidad Técnica Paricular de Loja]. Dspace UTPL. Obtenido de http://dspace.utpl.edu.ec/xmlui/bitstream/handle/123456789/14261/Angamarca_ Calderon_Silvio_Eduardo.pdf?sequence=1\&isAllowed=y

Arvidsson, R. (2019). On the use of ordinal scoring scales in social life cycle assessment. The International Journal of Life Cycle Assessment, 24(1), 604-606. doi:https://doi.org/10.1007/s11367-018-1557-2

Atmowardoyo, H. (2018). Research Methods in TEFL Studies: Descriptive Research, Case Study, Error Analysis, and R \& D. Journal of Language Teaching and Research, 9(1), 197-204. doi:http://dx.doi.org/10.17507/jltr.0901.25

Briones, W., Urquiza, L., Navia, J., \& Guevara, G. (2020). Asertividad comunicacional en los procesos académicos en época de pandemia en las Instituciones de Educación Superior. Journal of Science and Research, 5(1), 653-668. doi:https://doi.org/10.5281/zenodo.4441180

Calderón, L. (2017). Comunicación Asertiva y su Influencia en las Relaciones Interpersonales en la Unidad Educativa Martha Bucaram de Roldós[Tesis de pregrado, Universidad de Guayaquil]. Repositorio UG. Obtenido de http://repositorio.ug.edu.ec/bitstream/redug/21331/1/proyecto\%20de\%20comuni cacion\%20asertiva\%20tesis.pdf

Corrales, A., Quijano, N., \& Góngora, E. (2017). Empatía, Comunicación Asertiva y Seguimiento de Normas. Un programa para Desarrollar Habilidades para la Vida. Enseñanza e Investigación en Psicología, 22(1), 58-65. Obtenido de https://www.redalyc.org/pdf/292/29251161005.pdf 
Cortez, A. (2018). Indicadores de comunicación asertiva del docente y la generación del clima escolar en el aula en situaciones de aprendizaje [Tesis de maestría, Universidad Andina Simón Bolívar sede Ecuador]. Repositorio UASB. Obtenido de https://repositorio.uasb.edu.ec/bitstream/10644/6221/1/T2652-MIE-CortezIndicadores.pdf

Daniel, E. (2016). The Usefulness of Qualitative and Quantitative Approaches and Methods in Researching Problem-Solving Ability in Science Education Curriculum. Journal of Education and Practice, 7(15), 91-100. Obtenido de https://files.eric.ed.gov/fulltext/EJ1103224.pdf

Education Week. (15 de Octubre de 2019). Editorial Projects in Education. Obtenido de https://www.edweek.org/ew/section/multimedia/principals-heres-how-teachersview-you.html

Fabiani, B. (2017). Gobierno Electrónico y Desarrollo Institucional en las Universidades Públicas de la Zona 5, Ecuador [Tesis de doctorado, Universidad Nacional Mayor de San Marcos]. Cybertesis. Obtenido de https://cybertesis.unmsm.edu.pe/bitstream/handle/20.500.12672/8235/Fabiani_o b.pdf? sequence $=5 \&$ is Allowed $=\mathrm{y}$

Fernández, F. (2016). Comunicación Efectiva y Trabajo en Equipo (1era. ed.). Editorial Tutor Formación. Obtenido de https://es.scribd.com/document/341814940/Comunicacion-Efectiva-y-Trabajoen-Equipo-UF0346

Garbanzo, G. (2016). Desarrollo Organizacional y los Procesos de Cambio en las Instituciones Educativas, un Reto de la Gestión de la Educación. Revista Educación, 40(1), 67-87. doi:http://dx.doi.org/10.15517/revedu.v40i1.22534

Leal, M. (2019). La comunnicación asertiva como estrategia gerencial en el clima organizacional del LNB "Manuela Sáenz. [Tesis de maestría, Universidad de Carabobo]. mriuc. Obtenido de http://mriuc.bc.uc.edu.ve/bitstream/handle/123456789/8323/mleal.pdf?sequence $=1$

Leiva, M., \& Astorga, D. (2014). Condiciones del Desarrollo Institucional en los Centros Escolares de Chile. Revista Electrónica de Investigación Educativa, 16(2), 33-51. Obtenido de http://www.scielo.org.mx/pdf/redie/v16n2/v16n2a3.pdf 
Majid, U. (2018). Research Fundamentals: Study Design, Population, and Sample Size. URNCST Journal, 2(1), 1-7. doi:http://dx.doi.org/10.26685/urncst.16

Marotti, A., \& Pedroso, M. (2018). Applied Research Articles: narrowing the gap between research and organizations. Revista de Gestão, 25(4), 338-339. doi:https://doi.org/10.1108/REGE-10-2018-075

Mitchell, S., Loew, J., Millington, C., \& Dale, J. (2016). Providing end-of-life Care in General Practice: Findings of a National GP Questionnaire Survey. British Journal of General Practice, 66(650), 647-653. doi:https://dx.doi.org/10.3399\%2Fbjgp16X686113

Moscoso, V. (2016). Gerencia Académica y Desarrollo institucional del Instituto Tecnológico ESCA y propuesta de un Plan Estratégico con fines de Autoevaluación [Tesis de maestría, Universidad de Guayaquil]. Repositorio UG. Obtenido de http://repositorio.ug.edu.ec/bitstream/redug/3455/2/TESIS\%20MAE\%20NOV\% 202013.pdf

Organización de las Naciones Unidas para la Educación, la Ciencia y la Cultura. [UNESCO] . (2011). Manual de Gestión para Directores de Instituciones Educativas. Obtenido de https://www.academia.edu/29783946/Manual_de_Gestion_para_Directores_IE_ UNESCO_pdf

Pardo, C., \& Díaz, O. (2014). Desarrollo del Talento Humano como Factor Clave para el Desarrollo Organizacional, una Visión desde los Líderes de Gestión Humana en Empresas de Bogotá D.C. Eldesevier Doyma, 5(11), 39-48. doi:https://doi.org/10.1016/S2215-910X(14)70018-7

Ponto, J. (2015). Understanding and Evaluating Survey Research. JADPRO, 6(2), 168171. Obtenido de https://www.ncbi.nlm.nih.gov/pmc/articles/PMC4601897/

Rojas, O., Dolibeth, A., Mota, K., \& Quiñonez, J. (2020). El Liderazgo Transformacional desde la Perspectiva de la Pedagogía Humanista. Colección de Filosofía de la Educación, 28(1), 237-262. doi:https://doi.org/10.17163/soph.n28.2020.09

Román, Z. (2016). Comunicación Asertiva como Esencia en el Fortalecimiento de las Relaciones Interpersonales de los Docentes[Tesis de doctorado, Universidad 
Pedagógica Experimental]. Academia. Obtenido de https://www.academia.edu/31768368/Tesis_doctoral_nueva

Thomas, R. (2016). Nonexperimental Research: Strengths, Weaknesses and Issues of Precision. European Journal of Training and Development, 40(8), 676-690. Obtenido de https://www.emerald.com/insight/content/doi/10.1108/EJTD-072015-0058/full/html

Vargas, J., \& Guerra, E. (2016). Desarrollo Institucional como Instrumento para el Crecimiento Económico y el Desarrollo Social y Ambiental. Revista Conjeturas Sociológicas, $\quad 1(1), \quad 9-28 . \quad$ Obtenido de https://www.researchgate.net/publication/314174384_DESARROLLO_INSTIT UCIONAL_COMO_INSTRUMENTO_PARA_EL_CRECIMIENTO_ECONO MICO_Y_EL_DESARROLLO_SOCIAL_Y_AMBIENTAL

Zangirolami, J., Echeimberg, J., \& Leone, C. (2018). Research Methodology Topics: Cross-Sectional Studies. Journal of Human Growth and Development., 28(3), 356-360. doi:http://dx.doi.org/10.7322/jhgd.152198 